\title{
Universality in radiative corrections for non-supersymmetric heterotic vacua
}

\author{
Carlo Angelantonj* \\ Dipartimento di Fisica, Università di Torino and INFN Sezione di Torino \\ Via P. Giuria 1 - I-10125 Torino, Italy \\ E-mail: carlo.angelantonj@unito.it \\ loannis Florakis* \\ CERN Theory Division \\ 1211 Geneva 23, Switzerland \\ E-mail: ioannis.florakisecern.ch
}

\section{Mirian Tsulaia}

School of Physics M013, The University of Western Australia

35 Stirling Highway, Crawley W.A. 6009, Australia

E-mail: mirian.tsulaia@gmail.com

\begin{abstract}
Properties of moduli-dependent gauge threshold corrections in non-supersymmetric heterotic vacua are reviewed. In the absence of space-time supersymmetry these amplitudes are no longer protected and receive contributions from the whole tower of string states, BPS and not. Nevertheless, the difference of gauge thresholds for non-Abelian gauge groups displays a remarkable universality property, even when supersymmetry is absent. We present a simple heterotic construction that shares this universal behaviour and expose the necessary conditions on the supersymmetry breaking mechanism for universality to occur.
\end{abstract}

18th International Conference From the Planck Scale to the Electroweak Scale 25-29 May 2015

Ioannina, Greece

${ }^{*}$ Speaker. 


\section{Introduction}

A generic heterotic string vacuum is characterised by various gauge forces that typically do not combine into a simple gauge group. In fact, already at the string scale the gauge group factorises as $G=\prod_{\alpha} G_{\alpha}$. Nevertheless, the strength of the different gauge interactions is determined at three level by a single universal coupling, namely the string coupling constant ${ }^{1} g_{s}$.

This universality is however in principle spoiled at the quantum level, where the running of the gauge couplings strongly depends on the details of the spectrum of the charged particles. At the one-loop level one has the familiar expression

$$
\frac{16 \pi^{2}}{g_{\alpha}^{2}(\Lambda)}=\frac{16 \pi^{2}}{g_{s}^{2}}+\beta_{\alpha} \log \frac{M_{s}^{2}}{\Lambda^{2}}+\Delta_{\alpha}
$$

where the $\beta$-function coefficients $\beta_{\alpha}$ are determined by the (finite number of) light states only, while the infinite tower of massive string states computes the threshold corrections $\Delta_{\alpha}$. These are functions of the masses of the heavy states, and typically depend on the details of the compact space. Since the masses of states in the lower-dimensional spectrum can depend on the moduli of the internal manifold via their Kaluza-Klein and winding excitations, the $\Delta$ 's are actually functions on the moduli space of the string compactification.

In any quantum field theory with a finite number of degrees of freedom these functions are model dependent and thus the unification of gauge couplings at the quantum level is an issue that needs to be addressed independently for any vacuum of interest. String theory, however, possesses an infinite number of heavy states. It is well-known that this peculiarity highly constrains the full spectrum via the GSO projection (or modular invariance) and is actually responsible for the mild UV behaviour of string perturbation theory. It is thus reasonable to wonder whether it can also serve the purpose of constraining the perturbative expansion and yield quantum corrections which are in a sense universal and thus model independent.

Starting from the 90's, tremendous progress has been made in the analysis of quantum corrections to the low-energy effective action of heterotic vacua, and indeed a universal behaviour for the difference $\Delta_{\alpha \beta} \equiv \Delta_{\alpha}-\Delta_{\beta}$ of gauge thresholds in supersymmetric configurations has been discovered [1, 2, 3, 4]. A key role in this behaviour is played by supersymmetry. In particular, in supersymmetric vacua, the moduli dependence of the thresholds can only originate from sectors that preserve $\mathscr{N}=2$ supersymmetry and thus the associated one-loop amplitude is highly protected, receiving contributions only from the BPS spectrum. As a result, the pure stringy excitations contribute to the integrand holomorphically in the Teichmüller parameter $\tau$ and this, together with modular invariance and, thus, with the constraints coming from the infinite tower of heavy states, fixes uniquely the one-loop amplitudes, up to an overall additive constant, and universality is unavoidable.

What happens when supersymmetry is spontaneously broken or absent? Is universality still present or is the problem of gauge coupling unification an issue that must be addressed in a model dependent way?

Clearly, in order to be able to treat this problem in a fully-fledged string environment one needs to restrict the analysis to non-supersymmetric vacua which admit a solvable Conformal

\footnotetext{
${ }^{1}$ For simplicity, we assume here that all gauge groups are realised as level-one Kac-Moody algebras.
} 
Field Theory description. This is the case for the so-called coordinate dependent compactification $[5,6,7,8]$, i.e. a stringy realisation of the Scherk-Schwarz mechanism $[9,10]$. It amounts to shifting the masses of the perturbative states by their $R$-symmetry charges. More conveniently, this deformation can be reformulated at the world-sheet level in terms of freely-acting orbifolds ${ }^{2}$, where a supersymmetry breaking generator $g_{\mathrm{SB}}$ is coupled to a shift along one (or more) of the compact cycles. The supersymmetry breaking scale is thus related to the size of the compact cycles and supersymmetry can be recovered at the boundary of the moduli space. Whether or not this mechanism corresponds to a spontaneous breaking is an interesting open issue (see [13] for a related discussion).

In this case, the moduli dependent threshold corrections are no longer BPS saturated, and all string states do contribute to the running of the gauge couplings. As a result, the integrand is a genuine non-holomorphic function of $\tau$, and modular invariance, necessarily present in any consistent string vacuum, is no longer able to fix the amplitude uniquely.

Still, we have recently found $[14,15]$ that under some reasonable conditions on the supersymmetry breaking mechanism, an unexpected universal behaviour of $\Delta_{\alpha \beta}$ continues to hold. It is due to the presence of a spectral flow within the gauge degrees of freedom of the heterotic string, which now projects onto the bosonic (right moving) ground state. This is an important property for the phenomenology of non-supersymmetric heterotic vacua, a topic that has recently attracted renewed interest [13, 16, 17, 18, 19, 20, 21].

For concreteness we shall restrict our attention to compactifications of the ten-dimensional $E_{8} \times E_{8}$ heterotic string on the orbifolds $T^{6} / \Omega_{\mathrm{S}} \times \Omega_{\mathrm{SB}}$, where $\Omega_{\mathrm{S}}$ is some supersymmetry-preserving group while $\Omega_{\mathrm{SB}}$ is responsible for the complete breaking of supersymmetry and realises the freelyacting stringy Scherk-Schwarz mechanism. Since we are interested in the moduli dependence of the thresholds, $\Omega_{\mathrm{S}}$ must contain sectors which preserve $\mathscr{N}=2$ supersymmetry. For this reason, and without loss of generality, it suffices to restrict our attention to the cases where $\Omega_{\mathrm{S}}$ acts on a $T^{4}$ torus and realises the orbifold limit of the K3 surface. The supersymmetry breaking orbifold $\Omega_{\mathrm{SB}}$ is a priori unconstrained but, as we shall see, requirement of universality puts tight constraints on it.

Before moving to present the main results some comments are in order. Firstly, whenever supersymmetry is broken in string theory, one has to face the problem of classical (and quantum) stability of the vacuum. In fact, Scherk-Schwarz reductions in string theory usually induce tachyonic instabilities in some regions of moduli space, which are in a sense related to the Hagedorn instability of string thermodynamics. Although models which are classically stable at any point of moduli space have been constructed [22,23], and a link between the classical tachyons and properties of the tower of string excitations have been established [24, 25, 26], vacuum stability is still an open issue. In most cases the large-volume regime and/or the absence of Wilson lines guarantee a sensible perturbative analysis, or the blowing-up of orbifold singularities may turn the tachyonic states massive [16]. However, in general one has to be careful that Coleman-Weinberg-like potentials do not destabilise the vacuum. Secondly, low-scale supersymmetry breaking requires the size of the extra dimensions to be quite large. This observation can have some important consequences

\footnotetext{
${ }^{2} \mathrm{~A}$ recent discussion of the relation between freely-acting (a)symmetric orbifolds, Scherk-Schwarz gaugings of supergravity and non-geometric flux compactifications can be found in $[11,12]$.
} 
on the validity of the whole approach. Large volumes usually induce sizeable one-loop contributions to the vacuum energy, unless suitable cancellations occur [27, 28, 13, 29]. Similarly, they can induce large corrections to the running of the low-energy gauge couplings which invalidates the perturbative expansion $[30,29,31]$. A full control of these issues in a phenomenologically attractive scenario is still far from being achieved.

The paper is organised as follows. In section 2 we shall compute the threshold corrections for a simple, prototype model that manifests gauge threshold universality, while in section 3 we shall present the conditions that $\Omega_{\mathrm{SB}}$ must meet in order for universality to emerge. More details can be found in $[14,15,32]$.

\section{Gauge Threshold Universality: a simple example}

Let us consider the simple case of the $E_{8} \times E_{8}$ heterotic string compactified on the $T^{4} \times$ $T^{2} / \mathbb{Z}_{2} \times \mathbb{Z}_{2}$ orbifold. The first $\mathbb{Z}_{2}$ acts on the $T^{4}$ coordinates only and realises a singular limit of the $\mathrm{K} 3$ surface, thus preserving $\mathscr{N}=2$ supersymmetry in four dimensions. The second $\mathbb{Z}_{2}$ orbifold is instead responsible for the breaking of supersymmetry and its generator is given by $(-1)^{F+F_{1}+F_{2}} \delta$. Here $F$ is the space-time fermion number, $F_{1,2}$ assigns a minus sign to the spinorial representation inside $E_{8} \sim \operatorname{Spin}(16) / \mathbb{Z}_{2}$, while $\delta$ is an order-two shift along the horizontal side of the $T^{2}$ with Kähler and complex structure moduli $T$ and $U$. The one-loop partition function reads

$$
\begin{aligned}
& \mathscr{Z}=\frac{1}{2} \sum_{H, G=0}^{1} \frac{1}{2} \sum_{h, g=0}^{1}\left[\frac{1}{2} \sum_{a, b=0}^{1}(-)^{a+b} \vartheta\left[\begin{array}{l}
a / 2 \\
b / 2
\end{array}\right]^{2} \vartheta\left[\begin{array}{l}
a / 2+h / 2 \\
b / 2+g / 2
\end{array}\right] \vartheta\left[\begin{array}{l}
a / 2-h / 2 \\
b / 2-g / 2
\end{array}\right]\right] \\
& \times\left[\frac{1}{2} \sum_{k, \ell=0}^{1} \bar{\vartheta}\left[\begin{array}{l}
k / 2 \\
\ell / 2
\end{array}\right]^{6} \bar{\vartheta}\left[\begin{array}{l}
k / 2+h / 2 \\
\ell / 2+g / 2
\end{array}\right] \bar{\vartheta}\left[\begin{array}{l}
k / 2-h / 2 \\
\ell / 2-g / 2
\end{array}\right]\right]\left[\frac{1}{2} \sum_{r, s=0}^{1} \bar{\vartheta}\left[\begin{array}{l}
r / 2 \\
s / 2
\end{array}\right]^{8}\right] \\
& \times \frac{1}{\eta^{12} \bar{\eta}^{24}}(-)^{H(b+\ell+s)+G(a+k+r)+H G} \Gamma_{2,2}\left[\begin{array}{l}
H \\
G
\end{array}\right] \Lambda^{\mathrm{K} 3}\left[\begin{array}{l}
h \\
g
\end{array}\right],
\end{aligned}
$$

and is written in terms of the Dedekind $\eta$-function, the Jacobi theta constants $\vartheta$ with characteristics, and of the (twisted) lattices. In particular,

$$
\Gamma_{2,2}\left[\begin{array}{l}
H \\
G
\end{array}\right]=\tau_{2} \sum_{\vec{m}, \vec{n}} e^{i \pi G m_{1}} q^{\frac{1}{4 T_{2} U_{2}}\left|m_{2}-U m_{1}+\bar{T}\left(n^{1}+\frac{H}{2}+U n^{2}\right)\right|^{2}} \bar{q}^{\frac{1}{4 T_{2} U_{2}}\left|m_{2}-U m_{1}+T\left(n^{1}+\frac{H}{2}+U n^{2}\right)\right|^{2}}
$$

is the shifted Narain partition function associated to the freely-acted $T^{2}$, while the contributions of the world-sheet bosons along the $T^{4}$ is encoded into

$$
\Lambda^{\mathrm{K} 3}\left[\begin{array}{l}
h \\
g
\end{array}\right]=\left\{\begin{array}{cl}
\Gamma_{4,4} & \text { for }(h, g)=(0,0), \\
\frac{16|\eta|^{12}}{\left|\vartheta\left[\begin{array}{l}
1 / 2+h / 2 \\
1 / 2+g / 2
\end{array}\right] \vartheta\left[\begin{array}{l}
1 / 2-h / 2 \\
1 / 2-g / 2
\end{array}\right]\right|^{2}} & \text { for }(h, g) \neq(0,0),
\end{array}\right.
$$

with $\Gamma_{4,4}$ being the conventional Narain lattice associated to the $T^{4}$. The massless spectrum can be extracted from eq. (2.1) using standard techniques, and is not supersymmetric. In particular, the two gravitini of the $\mathrm{K} 3$ compactification acquire the mass

$$
m_{3 / 2}^{2}=\frac{|U|^{2}}{T_{2} U_{2}},
$$


that sets the scale of supersymmetry breaking. For a squared torus of radii $R_{1}$ and $R_{2}$ and vanishing $B$ field, the previous expression simply reduces to $m_{3 / 2}^{2}=1 / R_{1}^{2}$. The gauge sector includes non-Abelian vector bosons with gauge group $\mathrm{SO}(16) \times \mathrm{SO}(12)$. Notice that, as in the parent tendimensional $\mathrm{SO}(16) \times \mathrm{SO}(16)$ non-supersymmetry string [33,34], the spectrum is free of tachyonic excitations at the generic point of the $(T, U)$ moduli space. Tachyons are, however, expected to emerge if Wilson lines for the non-Abelian gauge group are turned on. We shall forbid this possibility in the present investigation, and work in regions of moduli space which are classically stable.

Contrary to standard $\mathscr{N}=2$ compactifications, the light spectrum charged with respect to the non Abelian gauge group depends on the position on the moduli space of the two-torus. This is due to the reversed GSO projection induced by the Scherk-Schwarz boost in the sector twisted by the susy breaking generator. As a result, the RNS vacuum survives the projection. It has non trivial windings and transforms in the bi-fundamental representation of $\mathrm{SO}(16) \times \mathrm{SO}(12)$,

$$
\mathrm{O}_{4} \mathrm{O}_{4} \bar{V}_{12} \bar{O}_{4} \bar{V}_{16} \times \frac{\Gamma_{2,2}\left[\begin{array}{l}
1 \\
0
\end{array}\right]-\Gamma_{2,2}\left[\begin{array}{l}
1 \\
1
\end{array}\right]}{2} .
$$

The mass of the lightest excitations, corresponding to vanishing momentum and windings along the second $T^{2}$ cycle, while carrying non-trivial momentum and winding numbers along the ScherkSchwarz cycle $m_{1}=2 n^{1}= \pm 1$, is given by

$$
m^{2}=\left|p_{\mathrm{R}}\right|^{2}=\frac{|U-T / 2|^{2}}{T_{2} U_{2}} .
$$

Therefore, these states can become massless at the point $U=T / 2$, that corresponds to $p_{\mathrm{R}}=0$. As we shall see, these states are responsible for the logarithmic divergence of the gauge thresholds.

Regardless of the presene or not of space-time supersymmetry, the one-loop heterotic thresholds $\Delta_{\alpha}$ are given by [35]

$$
\Delta_{\alpha}=\mathrm{R} . \mathrm{N} \cdot \int_{\mathscr{F}} d \mu \frac{i \tau_{2}}{\pi \eta^{2} \bar{\eta}^{2}} \sum_{a, b} \partial_{\tau}\left(\frac{\theta\left[\begin{array}{c}
a / 2 \\
b / 2
\end{array}\right]}{\eta}\right) \operatorname{Tr}_{\mathscr{H}\left[\begin{array}{l}
a \\
b
\end{array}\right]}\left[\left(Q_{\alpha}^{2}-\frac{1}{4 \pi \tau_{2}}\right) q^{L_{0}-c / 24} \bar{q}^{\bar{L}_{0}-\bar{c} / 24}\right]
$$

The trace runs over the Hilbert space $\mathscr{H}\left[\begin{array}{l}a \\ b\end{array}\right]$ of the internal $(c, \bar{c})=(9,22)$ CFT system with given spin structures, and is weighted with a Cartan charge $Q_{\alpha}$. The presence of $\tau_{2}^{-1}$, ascribed to a contact term in the correlation function of the Kac-Moody currents, is independent of the gauge group charges and can be associated to the universal coupling of the dilaton. The modular integral with measure $d \mu=\tau_{2}^{-2} d \tau_{1} d \tau_{2}$ is to be performed over the $\operatorname{SL}(2 ; \mathbb{Z})$ fundamental domain $\mathscr{F}$, and we invoke the modular-invariant regularisation prescription of $[36,37,38]$ to treat the infra-red divergences ascribed to the massless string states.

Because of the free action of the susy-breaking orbifold on the $T^{2}$, it is actually convenient to organise the contributions to the thresholds as

$$
\Delta_{\alpha}=\int_{\mathscr{F}} d \mu \sum_{H, G=0,1} \Delta_{\alpha}\left[\begin{array}{l}
H \\
G
\end{array}\right](T, U ; \tau)
$$

where each term in the sum refers to the $H$-twisted sector with the insertion of $g_{\mathrm{SB}}^{G}$ in the trace. Cleary, $\Delta_{\alpha}\left[\begin{array}{l}0 \\ 0\end{array}\right]$ corresponds to the $\mathscr{N}=2$ thresholds and thus is invariant under the full modular 
group. It is associated to a BPS amplitude where the RNS sector of the heterotic string is taken in its ground state. The remaining three terms are, instead, non-supersymmetric and receive contributions from the whole tower of string excitations. They are separately not modular invariant, but rather they form an $\operatorname{SL}(2 ; \mathbb{Z})$ orbit of $\Gamma_{0}(2)$ invariant expressions, where $\Gamma_{0}(2)$ is the Hecke congruence subgroup of order 2 . In fact,

$$
\Delta_{\alpha}\left[\begin{array}{l}
1 \\
0
\end{array}\right]=S \cdot \Delta_{\alpha}\left[\begin{array}{l}
0 \\
1
\end{array}\right] \quad \text { and } \quad \Delta_{\alpha}\left[\begin{array}{l}
1 \\
1
\end{array}\right]=T S \cdot \Delta_{\alpha}\left[\begin{array}{l}
0 \\
1
\end{array}\right]
$$

For this reason, it is convenient to partially unfold the non-supersymmetric contributions and trade the integral of $\Delta_{\alpha}\left[\begin{array}{l}0 \\ 1\end{array}\right]+\Delta_{\alpha}\left[\begin{array}{l}1 \\ 0\end{array}\right]+\Delta_{\alpha}\left[\begin{array}{l}1 \\ 1\end{array}\right]$ over the fundamental domain $\mathscr{F}$ for an integral of $\Delta_{\alpha}\left[\begin{array}{l}0 \\ 1\end{array}\right]$ only, but now over the larger $\Gamma_{0}(2)$ fundamental domain $\mathscr{F}_{2}$. As a result, eq. (2.8) reduces to

$$
\Delta_{\alpha}=\int_{\mathscr{F}} d \mu \Delta_{\alpha}\left[\begin{array}{l}
0 \\
0
\end{array}\right](T, U ; \tau)+\int_{\mathscr{F}_{2}} d \mu \Delta_{\alpha}\left[\begin{array}{l}
0 \\
1
\end{array}\right](T, U ; \tau) .
$$

Explicit computations for the case at hand yield

$$
\begin{aligned}
\Delta_{\mathrm{SO}(16)}\left[\begin{array}{l}
0 \\
0
\end{array}\right]= & -\frac{1}{24} \Gamma_{2,2}\left[\begin{array}{l}
0 \\
0
\end{array}\right] \frac{\hat{\bar{E}}_{2} \bar{E}_{4} \bar{E}_{6}-\bar{E}_{6}^{2}}{\bar{\eta}^{24}} \\
\Delta_{\mathrm{SO}(16)}\left[\begin{array}{l}
0 \\
1
\end{array}\right]= & -\frac{1}{576} \Gamma_{2,2}\left[\begin{array}{l}
0 \\
1
\end{array}\right] \frac{\Lambda^{K 3}\left[\begin{array}{l}
0 \\
0
\end{array}\right]}{\eta^{12} \bar{\eta}^{24}\left(\vartheta_{3}^{8}-\vartheta_{4}^{8}\right) \bar{\vartheta}_{3}^{4} \bar{\vartheta}_{4}^{4}\left[\left(\hat{\bar{E}}_{2}-\bar{\vartheta}_{3}^{4}\right) \bar{\vartheta}_{3}^{4} \bar{\vartheta}_{4}^{4}+8 \bar{\eta}^{12}\right]} \\
& -\frac{1}{48} \Gamma_{2,2}\left[\begin{array}{l}
0 \\
1
\end{array}\right] \frac{\bar{\vartheta}_{3}^{4} \bar{\vartheta}_{4}^{4}\left(\bar{\vartheta}_{3}^{4}+\bar{\vartheta}_{4}^{4}\right)\left[\left(\hat{\bar{E}}_{2}-\bar{\vartheta}_{3}^{4}\right) \bar{\vartheta}_{3}^{4} \bar{\vartheta}_{4}^{4}+8 \bar{\eta}^{12}\right]}{\bar{\eta}^{24}} \\
& -\frac{1}{72} \Gamma_{2,2}\left[\begin{array}{l}
0 \\
1
\end{array}\right] \frac{\vartheta_{2}^{4}\left(\vartheta_{3}^{8}-\vartheta_{4}^{8}\right)}{\eta^{12}} \frac{\left(\hat{\bar{E}}_{2}-\bar{\vartheta}_{3}^{4}\right) \bar{\vartheta}_{3}^{4} \bar{\vartheta}_{4}^{4}+8 \bar{\eta}^{12}}{\bar{\eta}^{12}}
\end{aligned}
$$

and

$$
\begin{aligned}
& \Delta_{\mathrm{SO}(12)}\left[\begin{array}{l}
0 \\
0
\end{array}\right]=-\frac{1}{24} \Gamma_{2,2}\left[\begin{array}{l}
0 \\
0
\end{array}\right] \frac{\hat{\bar{E}}_{2} \bar{E}_{4} \bar{E}_{6}-\bar{E}_{4}^{3}}{\bar{\eta}^{24}} \\
& \Delta_{\mathrm{SO}(12)}\left[\begin{array}{l}
0 \\
1
\end{array}\right]=-\frac{1}{576} \Gamma_{2,2}\left[\begin{array}{l}
0 \\
1
\end{array}\right] \frac{\Lambda^{K 3}\left[\begin{array}{l}
0 \\
0
\end{array}\right]}{\eta^{12} \bar{\eta}^{24}}\left(\vartheta_{3}^{8}-\vartheta_{4}^{8}\right) \bar{\vartheta}_{3}^{4} \bar{\vartheta}_{4}^{4}\left[\left(\hat{\bar{E}}_{2}-\bar{\vartheta}_{3}^{4}\right) \bar{\vartheta}_{3}^{4} \bar{\vartheta}_{4}^{4}+8 \bar{\eta}^{12}\right] \\
& -\frac{1}{48} \Gamma_{2,2}\left[\begin{array}{l}
0 \\
1
\end{array}\right] \frac{\bar{\vartheta}_{3}^{8} \bar{\vartheta}_{4}^{8}\left[\hat{\bar{E}}_{2}\left(\bar{\vartheta}_{3}^{4}+\bar{\vartheta}_{4}^{4}\right)+\bar{\vartheta}_{2}^{8}-2 \bar{\vartheta}_{3}^{4} \bar{\vartheta}_{4}^{4}\right]}{\bar{\eta}^{24}} \\
& -\frac{1}{72} \Gamma_{2,2}\left[\begin{array}{l}
0 \\
1
\end{array}\right]\left(\frac{\vartheta_{2}^{4}\left(\vartheta_{3}^{8}-\vartheta_{4}^{8}\right)}{\eta^{12}} \frac{\hat{\bar{E}}_{2} \bar{\vartheta}_{3}^{4} \bar{\vartheta}_{4}^{4}}{\bar{\eta}^{12}}\right. \\
& \left.+\frac{\vartheta_{2}^{4} \vartheta_{4}^{4}\left|\vartheta_{2}^{4}-\vartheta_{4}^{4}\right|^{2}-\vartheta_{2}^{4} \vartheta_{3}^{4}\left|\vartheta_{2}^{4}+\vartheta_{3}^{4}\right|^{2}}{\eta^{12} \bar{\eta}^{12}} \bar{\vartheta}_{3}^{4} \bar{\vartheta}_{4}^{4}\right)
\end{aligned}
$$

Aside from the holomorphic contributions $\Delta_{\mathrm{SO}(12)}\left[\begin{array}{l}0 \\ 0\end{array}\right]$ and $\Delta_{\mathrm{SO}(16)}\left[\begin{array}{l}0 \\ 0\end{array}\right]$ originating from the $\mathscr{N}=2$ supersymmetric sector, the remaining terms are manifestly non-holomorphic as a consequence of the breaking of supersymmetry. The first line in $\Delta_{\alpha}\left[\begin{array}{l}0 \\ 1\end{array}\right]$ is universal and depends on the hypermultiplet moduli of the $\mathrm{K} 3$ surface. The second line is again holomorphic, and indeed would correspond to a spontaneous $\mathscr{N}=4 \rightarrow \mathscr{N}=2$ breaking. It is BPS saturated, and is due to the simultaneours 
action of the two $\mathbb{Z}_{2}$ 's. Finally, the last contribution is again non-holomorphic and originates from sectors which are genuinely non-supersymmetric.

When taking the difference of gauge thresholds something surprising happens. As expected, the contact terms due to the universal dilaton exchange cancel out. Similarly, the contribution of the K3 hypermultiplets simplifies since it refers to a sector with trivial action of the supersymmetric $\mathbb{Z}_{2}$ orbifold which, thus, does not distinguish between the two gauge groups. What is completely not obvious is that also the remaining terms simplify, and yield a purely holomorphic contribution,

$$
\Delta_{\mathrm{SO}(16)}-\Delta_{\mathrm{SO}(12)}=-72 \int_{\mathscr{F}} d \mu \Gamma_{2,2}\left[\begin{array}{l}
0 \\
0
\end{array}\right]-\frac{1}{3} \int_{\mathscr{F}_{2}} d \mu \Gamma_{2,2}\left[\begin{array}{l}
0 \\
1
\end{array}\right]\left(\frac{\vartheta_{2}^{12}}{\eta^{12}}-8\right)
$$

This remarkable fact is a consequence of the the presence of MSDS-like spectral flow [39, 40, 41, 42] at work in the gauge sector of the heterotic string, according to which the combination

$$
\frac{\vartheta_{2}^{8}\left|\vartheta_{3}^{4}+\vartheta_{4}^{4}\right|^{2} \bar{\vartheta}_{3}^{4} \bar{\vartheta}_{4}^{4}-\vartheta_{2}^{4} \vartheta_{3}^{4}\left|\vartheta_{2}^{4}-\vartheta_{4}^{4}\right|^{2} \bar{\vartheta}_{3}^{4} \bar{\vartheta}_{4}^{4}+\vartheta_{2}^{4} \vartheta_{3}^{4}\left|\vartheta_{2}^{4}+\vartheta_{3}^{4}\right|^{2} \bar{\vartheta}_{3}^{4} \bar{\vartheta}_{4}^{4}}{\eta^{12} \bar{\eta}^{24}}
$$

is purely holomorphic $[14,15]$ ! As shown in [15], this unexpected property is not an accident of the specific model, but is actually the consequence of a more general universality identity for the differences of group traces

$$
\begin{gathered}
\left.\sum_{k, \ell, c, d=0,1}(-1)^{F[(k+c) G+(\ell+d) H]} \vartheta\left[\begin{array}{l}
k / 2 \\
\ell / 2
\end{array}\right] \vartheta^{6}\left[\begin{array}{l}
k / 2+h / 2 \\
\ell / 2+g / 2
\end{array}\right] \vartheta\left[\begin{array}{l}
k / 2-h / 2 \\
\ell / 2-g / 2
\end{array}\right] \vartheta\left[\begin{array}{l}
c / 2 \\
d / 2
\end{array}\right]^{8} \partial_{\tau} \log \frac{\vartheta^{[k / 2}\left[\begin{array}{l}
{[/ 2}
\end{array}\right]}{\vartheta^{c / 2}[/ 2}\right] \\
=-4 \pi i\left[4-16\left(\frac{1+(-1)^{F H}}{2}\right)\left(\frac{1+(-1)^{F G}}{2}\right)\right] \eta^{18} \vartheta\left[\begin{array}{l}
1 / 2+h / 2 \\
1 / 2+g / 2
\end{array}\right] \vartheta\left[\begin{array}{l}
1 / 2-h / 2 \\
1 / 2-g / 2
\end{array}\right]
\end{gathered}
$$

valid for $H, G, F \in \mathbb{Z}_{2}$ and for any $h, g$. This remarkable identity is a reflection of the general universality theorem that will be discussed in detail in the next section.

Returning to our threshold difference, and following [36, 37, 38, 43], one can explicitly evaluate the integrals to obtain $[14,15]$

$$
\begin{aligned}
\Delta_{\mathrm{SO}(16)}-\Delta_{\mathrm{SO}(12)=} & 72 \log \left[T_{2} U_{2}|\eta(T) \eta(U)|^{4}\right]-\frac{8}{3} \log \left[T_{2} U_{2}\left|\vartheta_{4}(T) \vartheta_{2}(U)\right|^{4}\right] \\
& +\frac{2}{3} \log \left|j_{\infty}(T / 2)-j_{\infty}(U)\right|^{4} .
\end{aligned}
$$

The terms in the first line are reminiscent of the threshold differences in supersymmetric vacua with hard and spontaneous $\mathscr{N}=4 \rightarrow \mathscr{N}=2$ breaking, and indeed a careful analysis shows that even in these non-supersymmetric vacua they are associated to the two supersymmetric sectors. The last term, instead, comes entirely from the non-supersymmetric (disconnected) orbit of this $\mathbb{Z}_{2} \times \mathbb{Z}_{2}$ orbifold, and the logarithmic singularity at $T / 2=U$ reflects the emergence of the extra massless states (2.5) at this point in moduli space. The coefficients have a clear field theoretical interpretation in terms of differences of $\beta$-function coefficients [15]. The function $j_{\infty}$ is the holomorphic invariant under $\Gamma_{0}(2)$ attached to the cusp $\infty$ and is related to the more familiar Dedekind function via $j_{\infty}(\tau)=24+(\eta(\tau) / \eta(2 \tau))^{24}$.

The surprising fact is that this simple behaviour of the gauge threshold differences is not particular to this $\mathbb{Z}_{2}$ orbifold, but is actually universal and extends to any compactification where the 
supersymmetry preserving $\mathbb{Z}_{2}$ group is replaced by any orbifold realisation of $\mathrm{K} 3$ or of any CalabiYau three-fold $[14,15]$. The only model-dependent quantities are the numerical coefficients in (2.16). In the next section, we shall formulate the necessary conditions that any heterotic vacuum, supersymmetric or not, needs to satisfy in order to display a universal behaviour of the gauge threshold differences.

\section{Gauge Threshold Universality: the general case}

As anticipated in the previous section, the gauge threshold corrections for a generic, tachyon free, heterotic vacuum are given by eq. (2.7). In this expression, the clearly non-holomorphic term originating from the insertion of $1 / \tau_{2}$ in the group trace, originating from the contact term of the two gauge-boson vertex operators, can be ascribed to dilaton exchange in a suitable degeneration limit of the loop diagram. Since the dilaton couples universally to any gauge group factor, the $1 / \tau_{2}$ contribution cancels out and thus, in a generic orbifold compactification without continuous Wilson lines, the difference of gauge thresholds takes the general form [15]

$$
\Delta_{\alpha \beta}=\int_{\mathscr{F}} d \mu \sum_{h, g} L\left[\begin{array}{l}
h \\
g
\end{array}\right](\tau) \bar{\Phi}\left[\begin{array}{l}
h \\
g
\end{array}\right](\bar{\tau}) \Gamma\left[\begin{array}{l}
h \\
g
\end{array}\right](G, B),
$$

where the sum runs over the various sectors of the orbifold. $L\left[\begin{array}{l}h \\ g\end{array}\right](\tau)$ is an holomorphic function of the modulus $\tau$ encoding the helicity super-trace over the left-moving sector, $\bar{\Phi}\left[\begin{array}{l}h \\ g\end{array}\right](\bar{\tau})$ is an antiholomorphic function encoding the $Q_{\alpha \beta}^{2}=Q_{\alpha}^{2}-Q_{\beta}^{2}$ graded trace over the right-moving sector, while $\Gamma\left[\begin{array}{l}h \\ g\end{array}\right](G, B)$ denotes a generic Narain lattice partition function associated to shifted tori with metric $G$ and $B$-field backgrounds.

In supersymmetric compactifications, the only moduli dependence of gauge thresholds originates from those sectors preserving $\mathscr{N}=2$ supersymmetry $^{3}$, which correspond to a $\mathrm{K} 3 \times T^{2}$ orbifold. These sectors have the remarkable property that their holomorphic contribution $L\left[\begin{array}{l}h \\ g\end{array}\right]$ drops to a constant, as a consequence of the BPS-ness of the $F_{\mu v} F^{\mu v}$ coupling. Technically, this property is the result of a cancellation between the helicity super-trace and the holomorphic contribution of the twisted K3 lattice, so that only the left-moving ground state survives. As a result, the moduli dependence of the threshold differences is encoded in

$$
\Delta_{\alpha \beta}=\int_{\mathscr{F}} d \mu \sum_{\substack{h, g=0 \\
(h, g) \neq(0,0)}}^{N-1} \bar{\Phi}\left[\begin{array}{l}
h \\
g
\end{array}\right](\bar{\tau}) \Gamma_{2,2}\left[\begin{array}{l}
h \\
g
\end{array}\right](T, U),
$$

where $\Gamma_{2,2}\left[\begin{array}{l}h \\ g\end{array}\right](T, U)$ is given by (2.2), and we allow for the possibility that the $\mathscr{N}=4 \rightarrow \mathscr{N}=2$ breaking be spontaneous, and realised via a freely acting orbifold.

The functions $\bar{\Phi}\left[\begin{array}{l}h \\ g\end{array}\right](\bar{\tau})$ are highly constrained by modular invariance, which is the rationale behind the celebrated universality of differences of gauge thresholds [1,3]. In fact, whenever the

\footnotetext{
${ }^{3}$ The untwisted unprojected sector of any supersymmetric orbifold compactification, although depending on the moduli of the six-dimensional torus, does not contribute to the threshold since it preserves the full $\mathscr{N}=4$ supersymmetry.
} 
lattice of the $T^{2}$ factorises, as in hard $\mathscr{N}=4 \rightarrow \mathscr{N}=2$ breaking without Wilson lines, it was realised that

$$
\bar{\Phi} \equiv \sum_{h, g} \bar{\Phi}\left[\begin{array}{l}
h \\
g
\end{array}\right](\bar{\tau})=\text { const }
$$

thus yielding the celebrated result in [1]. Indeed, in this case $\Phi$ is a holomorphic function invariant under the full $\operatorname{SL}(2 ; \mathbb{Z})$ modular group which is bound to be regular at the cusp $\tau=i \infty$. This last condition is the consequence of the fact that the untwisted bosonic vacuum $|0\rangle$ of the heterotic string is neutral with respect to the Kac-Moody currents while the charged twisted right-moving vacua $^{4}|n\rangle$ are not invariant under the orbifold action. Notice that this does not necessarily imply that each $\bar{\Phi}\left[\begin{array}{l}h \\ g\end{array}\right]$ be a constant for fixed $h$ and $g$. For instance, $\bar{\Phi}\left[\begin{array}{l}0 \\ 1\end{array}\right]$ is only invariant under the Hecke congruence subgroup $\Gamma_{0}(N)$, and the space of holomorphic invariant functions which are regular at the cusp $\tau=i \infty$ is much richer. In fact, one has the general decomposition

$$
\Phi\left[\begin{array}{l}
0 \\
1
\end{array}\right](\tau)=a+\sum_{\mathfrak{a} \neq \infty} b_{\mathfrak{a}} j_{\mathfrak{a}}(\tau)
$$

where $a$ and $b_{\mathfrak{a}}$ are constants and are the only model-dependent data. The index $\mathfrak{a}$ labels the various cusps of the fundamental domain $\mathscr{F}_{N}$ of $\Gamma_{0}(N)$, and $j_{\mathfrak{a}}$ are the invariant functions attached to the cusp $\mathfrak{a}[38,43]$.

The decomposition (3.5) dramatically extends the notion of universality in supersymmetric cases, when the two-dimensional lattice is shifted and couples to $\Phi\left[\begin{array}{l}0 \\ 1\end{array}\right]$. In fact, it is of crucial importance for the universality of related vacua with broken supersymmetry. To appreciate this point, let us focus for simplicity to the $\mathbb{Z}_{2}$ realisation of K3. Upon partially unfolding $\mathscr{F}$ [38], one can cast the integral (3.2) as

$$
\begin{aligned}
\Delta_{\alpha \beta} & =\int_{\mathscr{F}_{2}} d \mu \Gamma_{2,2}\left[\begin{array}{l}
0 \\
1
\end{array}\right](T, U) \bar{\Phi}\left[\begin{array}{l}
0 \\
1
\end{array}\right](\bar{\tau}) \\
& =a \int_{\mathscr{F}_{2}} d \mu \Gamma_{2,2}\left[\begin{array}{l}
0 \\
1
\end{array}\right](T, U)+b_{0} \int_{\mathscr{F}_{2}} d \mu \Gamma_{2,2}\left[\begin{array}{l}
0 \\
1
\end{array}\right](T, U) \bar{J}_{0}(\bar{\tau}) .
\end{aligned}
$$

The first integral was evaluated in $[2,46,38]$ while the the second one was evaluated in $[38,43]$ and, for a momentum shift along the first cycle of $T^{2}$, read

$$
\Delta_{\alpha \beta}=-\left(a+24 b_{0}\right) \log \left[T_{2} U_{2}\left|\vartheta_{4}(T) \vartheta_{2}(U)\right|^{4}\right]-2 b_{0} \log \left|j_{\infty}(T / 2)-j_{\infty}(U)\right|^{4} .
$$

Similar expressions can be obtained also for the other orbifold realisations of K3. While the first contribution is regular in the bulk of the $(T, U)$ moduli space, the second one displays a logarithmic singularity when $T / 2=U$, plus all their $\Gamma_{0}(2)_{U}$ images. Therefore, a non-vanishing $b_{0}$ coefficient

\footnotetext{
${ }^{4}$ Among the unphysical states particularly important for our investigation are those associated to the vacuum of the right moving sector. These include the ubiquitous untwisted uncharged bosonic vacuum $|0\rangle$ of the heterotic string, as well as the vacua

$$
|n\rangle \sim \bar{\sigma}_{n} \bar{\chi}_{n}|0\rangle, \quad n=1, \ldots, N-1,
$$

associated to the right-moving ground states of the $g^{n}$ twisted sectors. $\sigma_{n}$ is the standard bosonic twist field associated to the right-moving twisted K3 coordinates and has conformal weight $\Delta_{\sigma}=\frac{n}{N}\left(1-\frac{n}{N}\right)$, whereas $\chi_{n}$ is the fermionic twist field associated to the "twisted Kac-Moody current" and has $\mathrm{U}(1)$ charge $Q_{\chi}= \pm \frac{n}{N}$ and conformal weight $\Delta_{\chi}=Q_{\chi}^{2}$ $[44,45]$. We stress once more that $|0\rangle$ is neutral with respect to the $\mathrm{E}_{8} \times \mathrm{E}_{8}$ Cartan charges, while $|n\rangle$ always carries non-trivial charge with respect to one of these Cartan's, at least in the case of standard embedding.
} 
is associated to the presence of extra massless states at these special points. The lesson to be learnt from this discussion, is that the presence of extra charged massless states, whether they be hypermultiplets or vector multiplets, significantly modifies the standard notion of universality of $\Delta_{\alpha \beta}$ and extends it with the contribution in eq. (3.7).

As we shall see momentarily, this very same universal behaviour of supersymmetric gauge thresholds also holds in heterotic models with spontaneously broken supersymmetry [14, 15], provided one considers thresholds for groups of rank larger than one, and certain specific conditions are satisfied by the supersymmetry-breaking generators. To this end, we focus on heterotic vacua on $T^{6} / \Omega_{\mathrm{S}} \times \Omega_{\mathrm{SB}}$, where $\Omega_{\mathrm{S}}$ is a supersymmetric orbifold, while $\Omega_{\mathrm{SB}}$ has a free action, and is responsible for the spontaneous breaking of supersymmetry.

The main difference with the supersymmetric case, is that now the $F_{\mu v} F^{\mu v}$ coupling is no longer BPS protected, and receives contributions from the whole tower of string states. This implies that there are sectors where the helicity supertrace no longer cancels against the contribution of the twisted lattice, and therefore the $L\left[\begin{array}{l}h \\ g\end{array}\right]$ 's are no longer constants for all $h$ and $g$. As a result, aside from lattice contributions, the integrand in the threshold modular integral is no longer holomorphic, and modular invariance fails to constrain it uniquely. Universality is recovered whenever the $\Phi\left[\begin{array}{l}h \\ g\end{array}\right]$ are constants for suitable values of $h$ and $g$, so that the product $L\left[\begin{array}{l}h \\ g\end{array}\right] \Phi\left[\begin{array}{l}h \\ g\end{array}\right]$ reduces to a holomorphic function. In this case modular invariance fixes the $\tau$ dependence of the (lattice independent) integrand up to few model dependent coefficients.

To be concrete, let us give a closer look at the conditions required for universality. We denote by $g_{\mathrm{S}}$ and $g_{\mathrm{SB}}$ generic elements of the orbifold groups $\Omega_{\mathrm{S}}$ and $\Omega_{\mathrm{SB}}$. Clearly, $L\left[\begin{array}{l}g_{\mathrm{S}}^{\prime} \\ g_{\mathrm{S}}\end{array}\right]$ is constant due to the effective supersymmetry present in this orbit, and therefore yields only universal contributions to the thresholds. However, in supersymmetry-breaking orbits $L\left[\begin{array}{l}g \mathrm{~S} \\ g_{\mathrm{SB}}\end{array}\right]$ is no longer a constant and thus universality is lost unless $\Phi\left[\begin{array}{c}g_{\mathrm{S}} \\ g_{\mathrm{SB}}\end{array}\right]$ is. In this case, modular invariance leads to the decomposition of $L\left[\begin{array}{c}g_{\mathrm{S}} \\ g_{\mathrm{SB}}\end{array}\right]$ into functions invariant under some finite index subgroup of $\mathrm{SL}(2 ; \mathbb{Z})$, analogously to eq. (3.5), and universality is restored.

Which are the conditions required for this to occur? In other words, for which choices of $\Omega_{\mathrm{SB}}$ are the $\Phi\left[\begin{array}{c}g_{\mathrm{S}} \\ g_{\mathrm{SB}}\end{array}\right]$ constant? In order to answer this question, let us recall that a generic orbifold element $g$ involves a separate action on the left and on the right moving degrees of freedom, and thus is decomposable as $g=\gamma^{\mathrm{L}} \otimes \gamma^{\mathrm{R}}$. In particular, $\gamma^{\mathrm{L}}$ involves the action on the RNS sector and is the only potential source for supersymmetry breaking. Of course, $\gamma^{\mathrm{R}}$ is not arbitrary but is correlated to $\gamma^{\mathrm{L}}$ by modular invariance of the one-loop partition function, and typically involves an action on the gauge degrees of freedom. We shall assume that the orbifold acts left-right symmetrically on the six compact coordinates. As a result, $\gamma^{\mathrm{L}}$ determines the $L\left[\begin{array}{l}h \\ g\end{array}\right]$ 's and, most importantly, $\gamma^{\mathrm{R}}$ determines the $\Phi\left[\begin{array}{l}h \\ g\end{array}\right]$ 's. This simple observation allows one to find the necessary conditions for universality to hold. Denote by $g_{\mathrm{SB}}=\gamma_{\mathrm{SB}}^{\mathrm{L}} \otimes \gamma^{\mathrm{R}}$ the decomposition of the supersymmetry breaking generators of $\Omega_{\mathrm{SB}}$. If one can replace $\gamma_{\mathrm{SB}}^{\mathrm{L}}$ with a new supersymmetry preserving action $\tilde{\gamma}_{\mathrm{S}}^{\mathrm{L}}$, while keeping the same action $\gamma^{\mathrm{R}}$ on the right-movers

$$
g_{\mathrm{SB}}=\gamma_{\mathrm{SB}}^{\mathrm{L}} \otimes \gamma^{\mathrm{R}} \rightarrow \tilde{g}_{\mathrm{S}}=\tilde{\gamma}_{\mathrm{S}}^{\mathrm{L}} \otimes \gamma^{\mathrm{R}},
$$

in a way that leads to a consistent supersymmetric string model, then the $\Phi\left[\begin{array}{l}g \mathrm{~S} \\ g_{\mathrm{SB}}\end{array}\right]$ 's are again decomposed as in (3.5). Therefore, in the absence of extra charged massless states in the supersymmetric 
"relative" model twisted by $\Omega_{\mathrm{S}} \times \tilde{\Omega}_{\mathrm{S}}$, the $b_{\mathfrak{a}}$ coefficients vanish and one indeed recovers universality. We can summarise the above in the

Universality Theorem: Any non-supersymmetric heterotic orbifold $T^{6} / \Omega_{\mathrm{S}} \times \Omega_{\mathrm{SB}}$ yields a universal behaviour in the difference of gauge thresholds $\Delta_{\alpha \beta}$ for gauge groups $G_{\alpha}$ and $G_{\beta}$, of rank larger than one, if $\Omega_{\mathrm{SB}}$ can be consistently replaced by a supersymmetric orbifold $\tilde{\Omega}_{\mathrm{S}}$ with the very same action on the right-moving degrees of freedom, and provided no extra massless states charged with respect to $G_{\alpha} \times G_{\beta}$ emerge in the bulk of the moduli space of the supersymmetric orbifold $T^{6} / \Omega_{\mathrm{S}} \times \tilde{\Omega}_{\mathrm{S}}$.

Which are the allowed possibilities for $\Omega_{\mathrm{SB}}$ ? Recall that in symmetric orbifold constructions, fixing the action on the right moving sector automatically determines the action on the left movers as well. Therefore, the latter is either compatible with supersymmetry or not, and there is no possibility to find a $\tilde{\gamma}_{\mathrm{S}}^{\mathrm{L}}$ that turns $\Omega_{\mathrm{SB}}$ to $\tilde{\Omega}_{\mathrm{S}}$. A careful study [15] of various possibilities leads then to the following

Corollary: The non-supersymmetric heterotic orbifolds $T^{6} / \Omega_{\mathrm{S}} \times \Omega_{\mathrm{SB}}$ that yield a universal behaviour in the difference of gauge thresholds $\Delta_{\alpha \beta}$, for gauge groups $G_{\alpha}$ and $G_{\beta}$ of rank greater than one, are of the form

$$
\Omega_{\mathrm{SB}}=(-1)^{F} \delta \gamma_{\text {gauge }},
$$

with the action on the complex fermions $\tilde{\psi}^{a}(\bar{z})$ realising the $E_{8} \times E_{8}$ Kac-Moody currents $\tilde{J}^{a b}(\bar{z})=$ $i \tilde{\psi}^{a} \tilde{\psi}^{b}(\bar{z})$ at the factorised point, chosen to have the eigenvalues $\gamma_{\text {gauge }}=e^{2 \pi i \lambda_{\text {gauge }}}$

$$
\lambda_{\text {gauge }}:\left(0^{8} ; 0^{8}\right) \quad, \quad\left(1,0^{7} ; 1,0^{7}\right) \quad, \quad\left((1 / 2)^{2}, 0^{6} ;(1 / 2)^{2}, 0^{6}\right) .
$$

In these three cases, the universal form of threshold differences for non-abelian gauge groups is

$$
\begin{gathered}
\Delta_{\alpha \beta}=\sum_{i=1,2,3} a_{i} \log \left[T_{2}^{(i)} U_{2}^{(i)}\left|\eta\left(T^{(i)}\right) \eta\left(U^{(i)}\right)\right|^{4}\right]+b_{i} \log \left[T_{2}^{(i)} U_{2}^{(i)}\left|\vartheta_{4}\left(T^{(i)}\right) \vartheta_{2}\left(U^{(i)}\right)\right|^{4}\right] \\
+c_{i} \log \left|j_{\infty}\left(T^{(i)} / 2\right)-j_{\infty}\left(U^{(i)}\right)\right|^{4} .
\end{gathered}
$$

The constants $a_{i}, b_{i}$ and $c_{i}$ are model dependent and can be computed by the knowledge of the massless spectrum alone.

This result extends the notion of universality, familiar from supersymmetric constructions, to the case of heterotic vacua with (spontaneously) broken supersymmetry. The analysis of rankone gauge group factors is more involved. One may show that the universal expression (3.11) is modified by an additional non-universal contribution which, however, is exponentially suppressed with the volume of $T^{2}$. Universality is, therefore, asymptotically restored in the large $T_{2}$ limit and we refer the interested reader to [15] for more details. Significantly different is the case of models which violate the requirements of the above universality theorem. A careful analysis reveals that in such situations universality is unequivocably lost and cannot be restored even in large volume limits.

We would like to conclude the discussion with a comment on issues of chirality. Clearly, the Scherk-Schwarz mechanism seen as a freely-acting orbifold does not generate any fixed points. 
As a result, the only way for non-supersymmetric models with universality to possess also a chiral spectrum of charged matter, is for the parent supersymmetric model generated by $\Omega_{\mathrm{S}}$ to already have this property. Given that the $\Omega_{\mathrm{SB}}$ orbifold does not distinguish between chirality assignments of spinor representations of the gauge group, one is led to conclude that the resulting non-supersymmetric model built out of $\Omega_{\mathrm{S}} \times \Omega_{\mathrm{SB}}$ will indeed contain chiral matter, provided the conditions of the universality theorem are met. An explicit example of such chiral constructions with universality has been given in [15].

\section{Acknowledgements}

C.A. and I.F. would like to thank the organisers of the Planck ' 15 conference for their kind invitation to present this work. The work of C.A. has been partially supported by the Compagnia di San Paolo contract "Modern Application in String Theory" (MAST) TO-Call3-2012-0088. The work of M.T. has been partially supported by the grant 31/89 of the Shota Rustaveli National Science Foundation.

\section{References}

[1] L. J. Dixon, V. Kaplunovsky and J. Louis, "Moduli dependence of string loop corrections to gauge coupling constants," Nucl. Phys. B 355 (1991) 649.

[2] P. Mayr and S. Stieberger, "Threshold corrections to gauge couplings in orbifold compactifications," Nucl. Phys. B 407 (1993) 725 [hep-th/9303017].

[3] E. Kiritsis, C. Kounnas, P. M. Petropoulos and J. Rizos, "Universality properties of $\mathscr{N}=2$ and $\mathscr{N}=1$ heterotic threshold corrections," Nucl. Phys. B 483 (1997) 141 [hep-th/9608034].

[4] H. P. Nilles and S. Stieberger, "String unification, universal one loop corrections and strongly coupled heterotic string theory," Nucl. Phys. B 499 (1997) 3 doi:10.1016/S0550-3213(97)00315-5 [hep-th/9702110].

[5] R. Rohm, "Spontaneous Supersymmetry Breaking in Supersymmetric String Theories," Nucl. Phys. B 237 (1984) 553.

[6] C. Kounnas and M. Porrati, "Spontaneous Supersymmetry Breaking In String Theory," Nucl. Phys. B 310 (1988) 355.

[7] S. Ferrara, C. Kounnas, M. Porrati and F. Zwirner, "Superstrings With Spontaneously Broken Supersymmetry And Their Effective Theories," Nucl. Phys. B 318 (1989) 75.

[8] C. Kounnas and B. Rostand, "Coordinate Dependent Compactifications and Discrete Symmetries," Nucl. Phys. B 341 (1990) 641.

[9] J. Scherk and J. H. Schwarz, "Spontaneous Breaking of Supersymmetry Through Dimensional Reduction,” Phys. Lett. B 82 (1979) 60.

[10] J. Scherk and J. H. Schwarz, "How to Get Masses from Extra Dimensions," Nucl. Phys. B 153 (1979) 61. 
[11] C. Condeescu, I. Florakis and D. Lust, "Asymmetric Orbifolds, Non-Geometric Fluxes and Non-Commutativity in Closed String Theory,” JHEP 1204 (2012) 121 doi:10.1007/JHEP04(2012)121 [arXiv:1202.6366 [hep-th]].

[12] C. Condeescu, I. Florakis, C. Kounnas and D. Lust, "Gauged supergravities and non-geometric Q/R-fluxes from asymmetric orbifold CFT‘s,” JHEP 1310 (2013) 057 doi:10.1007/JHEP10(2013)057 [arXiv:1307.0999 [hep-th]].

[13] S. Abel, K. R. Dienes and E. Mavroudi, "Towards a nonsupersymmetric string phenomenology," Phys. Rev. D 91 (2015) 12, 126014 doi:10.1103/PhysRevD.91.126014 [arXiv:1502.03087 [hep-th]].

[14] C. Angelantonj, I. Florakis and M. Tsulaia, "Universality of Gauge Thresholds in Non-Supersymmetric Heterotic Vacua," Phys. Lett. B 736 (2014) 365 doi:10.1016/j.physletb.2014.08.001 [arXiv:1407.8023 [hep-th]].

[15] C. Angelantonj, I. Florakis and M. Tsulaia, "Generalised universality of gauge thresholds in heterotic vacua with and without supersymmetry," Nucl. Phys. B 900 (2015) 170 doi:10.1016/j.nuclphysb.2015.09.007 [arXiv:1509.00027 [hep-th]].

[16] M. Blaszczyk, S. Groot Nibbelink, O. Loukas and S. Ramos-Sanchez, "Non-supersymmetric heterotic model building,” JHEP 1410 (2014) 119 [arXiv:1407.6362 [hep-th]].

[17] A. Lukas, Z. Lalak and E. E. Svanes, "Heterotic Moduli Stabilisation and Non-Supersymmetric Vacua,” JHEP 1508 (2015) 020 [arXiv:1504.06978 [hep-th]].

[18] J. M. Ashfaque, P. Athanasopoulos, A. E. Faraggi and H. Sonmez, "Non-Tachyonic Semi-Realistic Non-Supersymmetric Heterotic String Vacua," arXiv:1506.03114 [hep-th].

[19] M. Blaszczyk, S. G. Nibbelink, O. Loukas and F. Ruehle, "Calabi-Yau compactifications of non-supersymmetric heterotic string theory," arXiv:1507.06147 [hep-th].

[20] S. G. Nibbelink, O. Loukas and F. Ruehle, “(MS)SM-like models on smooth Calabi-Yau manifolds from all three heterotic string theories," arXiv:1507.07559 [hep-th].

[21] Y. Satoh, Y. Sugawara and T. Wada, "Non-supersymmetric Asymmetric Orbifolds with Vanishing Cosmological Constant,” arXiv:1512.05155 [hep-th].

[22] C. Angelantonj, M. Cardella and N. Irges, “An Alternative for Moduli Stabilisation,” Phys. Lett. B 641 (2006) 474 [hep-th/0608022].

[23] C. Angelantonj, C. Kounnas, H. Partouche and N. Toumbas, Nucl. Phys. B 809 (2009) 291 doi:10.1016/j.nuclphysb.2008.10.010 [arXiv:0808.1357 [hep-th]].

[24] D. Kutasov and N. Seiberg, "Number of degrees of freedom, density of states and tachyons in string theory and CFT,” Nucl. Phys. B 358 (1991) 600.

[25] K. R. Dienes, "Modular invariance, finiteness, and misaligned supersymmetry: New constraints on the numbers of physical string states," Nucl. Phys. B 429 (1994) 533 [hep-th/9402006].

[26] C. Angelantonj, M. Cardella, S. Elitzur and E. Rabinovici, "Vacuum stability, string density of states and the Riemann zeta function," JHEP 1102 (2011) 024 [arXiv:1012.5091 [hep-th]].

[27] J. A. Harvey, "String duality and nonsupersymmetric strings," Phys. Rev. D 59 (1999) 026002 [hep-th/9807213].

[28] C. Angelantonj, I. Antoniadis and K. Forger, "Nonsupersymmetric type I strings with zero vacuum energy," Nucl. Phys. B 555 (1999) 116 [hep-th/9904092]. 
[29] C. Kounnas and H. Partouche, "Stringy $\mathscr{N}=1$ super no-scale models," arXiv:1511.02709 [hep-th].

[30] A. E. Faraggi, C. Kounnas and H. Partouche, "Large volume susy breaking with a solution to the decompactification problem," Nucl. Phys. B 899 (2015) 328 [arXiv:1410.6147 [hep-th]].

[31] H. Partouche, "Large volume supersymmetry breaking without decompactification problem," arXiv: 1601.04564 [hep-th].

[32] I. Florakis, "Universality of radiative corrections to gauge couplings for strings with spontaneously broken supersymmetry,” J. Phys. Conf. Ser. 631 (2015) 1, 012079 doi:10.1088/1742-6596/631/1/012079 [arXiv:1502.07537 [hep-th]].

[33] L. Alvarez-Gaume, P. H. Ginsparg, G. W. Moore and C. Vafa, "An O $(16) \times \mathrm{O}(16)$ Heterotic String," Phys. Lett. B 171 (1986) 155.

[34] L. J. Dixon and J. A. Harvey, "String Theories in Ten-Dimensions Without Space-Time Supersymmetry," Nucl. Phys. B 274 (1986) 93.

[35] V. S. Kaplunovsky, “One Loop Threshold Effects in String Unification,” Nucl. Phys. B 307 (1988) 145 [Nucl. Phys. B 382 (1992) 436] [hep-th/9205068].

[36] C. Angelantonj, I. Florakis and B. Pioline, "A new look at one-loop integrals in string theory," Commun. Num. Theor. Phys. 6 (2012) 159 [arXiv:1110.5318 [hep-th]].

[37] C. Angelantonj, I. Florakis and B. Pioline, “One-Loop BPS amplitudes as BPS-state sums,” JHEP 1206 (2012) 070 [arXiv:1203.0566 [hep-th]].

[38] C. Angelantonj, I. Florakis and B. Pioline, "Rankin-Selberg methods for closed strings on orbifolds," JHEP 1307 (2013) 181 [arXiv:1304.4271 [hep-th]].

[39] C. Kounnas, "Massive Boson-Fermion Degeneracy and the Early Structure of the Universe," Fortsch. Phys. 56 (2008) 1143 [arXiv:0808.1340 [hep-th]].

[40] I. Florakis and C. Kounnas, "Orbifold Symmetry Reductions of Massive Boson-Fermion Degeneracy,” Nucl. Phys. B 820 (2009) 237 [arXiv:0901.3055 [hep-th]].

[41] I. Florakis, C. Kounnas and N. Toumbas, "Marginal Deformations of Vacua with Massive boson-fermion Degeneracy Symmetry,” Nucl. Phys. B 834 (2010) 273 [arXiv:1002.2427 [hep-th]].

[42] A. E. Faraggi, I. Florakis, T. Mohaupt and M. Tsulaia, "Conformal Aspects of Spinor-Vector Duality," Nucl. Phys. B 848 (2011) 332 [arXiv:1101.4194 [hep-th]].

[43] C. Angelantonj, I. Florakis and B. Pioline, "Threshold corrections, generalised prepotentials and Eichler integrals," Nucl. Phys. B 897 (2015) 781 [arXiv:1502.00007 [hep-th]].

[44] L. J. Dixon, D. Friedan, E. J. Martinec and S. H. Shenker, "The Conformal Field Theory of Orbifolds," Nucl. Phys. B 282 (1987) 13.

[45] S. Hamidi and C. Vafa, "Interactions on Orbifolds," Nucl. Phys. B 279 (1987) 465.

[46] E. Kiritsis, C. Kounnas, P. M. Petropoulos and J. Rizos, "String threshold corrections in models with spontaneously broken supersymmetry," Nucl. Phys. B 540 (1999) 87 [hep-th/9807067]. 